CARTA AL EDITOR

\section{POSITIVIDAD REAL, FALSA \\ NEGATIVIDAD Y OMISIÓN DIAGNÓSTICA DE INFECCIÓN SARS-COV-2 EN PERÚ}

\section{TRUE POSITIVITY, FALSE NEGATIVITY AND DIAGNOSTIC OMISSION OF SARS-COV-2 INFECTION IN PERU}

\section{Oscar J. Mújica(101,a,b,c, Luis Suárez-Ognio(iD2,a,c}

1 Departamento de Evidencia e Inteligencia para la Acción en Salud Organización Panamericana de la Salud/Organización Mundial de la Salud, Washington DC, Estados Unidos.

2 Escuela de Medicina, Universidad Peruana de Ciencias Aplicadas, Lima, Perú.

a Médico cirujano; ${ }^{\mathrm{b}}$ magister en Salud Pública; ${ }^{\mathrm{c}}$ especialista en Epidemiología de Campo.

Sr. Editor: Según las cifras oficiales del Ministerio de Salud, al 16 de julio del año en curso - exactamente 133 días o 19 semanas completas desde la confirmación del primer caso de enfermedad por coronavirus (COVID-19) en el país-, se han procesado las muestras de 2003963 personas para diagnosticar la infección por SARS-CoV-2, el agente causal de la COVID-19, y se reportaron 341586 resultados positivos (positividad observada: 17,0\%). Del total de muestras procesadas, 309746 $(15,5 \%)$ corresponden a pruebas moleculares (RT-PCR) y las restantes a pruebas serológicas «rápidas»; el 26,4\% (90 047) de los resultados positivos a la COVID-19 proviene de las pruebas moleculares procesadas ${ }^{(1)}$.

A partir de la información presentada, actualizada a diario por el Ministerio de Salud, nos propusimos evaluar la validez diagnóstica para el conjunto de la población testeada, estimando la positividad real (Pr), falsa negatividad (FN) y omisión diagnóstica (OD). Para ello, resolvimos una matriz de confusión por cada tipo de prueba diagnóstica de SARSCoV-2 aplicando a la positividad observada (Po) un conjunto de valores de sensibilidad (S) y especificidad (E) típicos a cada prueba (incluyendo sus intervalos de confianza para estimar el rango de credibilidad de resultados) mediante la fórmula de Ahlbom-Norell ${ }^{(2)}$ :

$$
\operatorname{Pr}=\frac{\operatorname{Po}+\mathrm{E}-1}{\mathrm{~S}+\mathrm{E}-1}
$$

Citar como: Mújica OJ, Suárez-Ognio L. Positividad real, falsa negatividad y omisión diagnóstica de infección SARS-CoV-2 en Perú. Rev Peru Med Exp Salud Publica. 2020;37(4):779-80. doi: https://doi.org/10.17843/rpmesp.2020.374.6205.

Correspondencia: Oscar J Mújica; 525 23rdSt. N.W. Washington DC 20037. United States of America; mujicaos@paho.org

Recibido: 21/07/2020 Aprobado: 16/09/2020 En línea: 05/11/2020
Específicamente, asumimos S = 89\% (IC95\%: 81\% - 94\%) ${ }^{(3)}$ y $\mathrm{E}=98 \%$ (IC95\%: 97\% - 99\%) ${ }^{(4)}$ para pruebas moleculares, $\mathrm{y}$ $\mathrm{S}=72,2 \%$ (IC95\%: 63,5\% - 79,5\%) y $\mathrm{E}=98 \%$ (IC95\%: 92,9\% $99,8 \%)^{(5)}$ para pruebas serológicas, considerando para el periodo estudiado un tiempo promedio de retraso de 9 a 11 días entre el inicio de síntomas y el reporte de resultado de la prueba diagnóstica ${ }^{(6)}$.

Los hallazgos de nuestra evaluación muestran que, entre los dos millones de personas testeadas, existen 406431 casos de infección por SARS-CoV-2 (positividad real: 20,28\%; rango de credibilidad, RC: 20,23\% - 20,34\%); entre los resultados reportados como positivos (341 586) existen 31951 (RC: 4925 - 109 848) falsos positivos; entre los resultados reportados como negativos (1 662 377) existen 96796 (RC: 69759 - 105 155) falsos negativos. Estos resultados coinciden con una razón de omisión diagnóstica (OD) igual a 3,20 (RC: 2,20 - 4,83) (Figura 1).

Aunque la discrepancia entre la positividad observada (la que se estima por el número de pruebas con resultado positivo sobre el total de pruebas realizadas) y la positividad real (la que refleja la verdadera prevalencia de infección en el universo testeado) es esperable debido a la inherente imperfección de las pruebas diagnósticas, en un escenario de propagación epidémica la magnitud de esta discrepancia puede ser determinante en la efectividad de las medidas de control y la eficacia de la contención epidémica. Para los niveles de sensibilidad y especificidad asumidos, concordante con lo reportado en una exhaustiva revisión sistemática Cochrane ${ }^{(5)}$, nuestros hallazgos muestran que, entre el total de personas testadas, por cada 3 verdaderas infectadas por SAR-CoV-2 existe, en promedio, una persona infectada no detectada; una razón de omisión diagnóstica que se mantiene relativamente estable en el periodo evaluado (material suplementario).

La elevada falsa negatividad observada entre la población testeada en el país está mayormente generada por la baja validez diagnóstica, especialmente la sensibilidad subóptima, de las pruebas serológicas, que en el universo estudiado constituyen el $85 \%$ de las pruebas diagnósticas administradas. La razón de omisión diagnóstica encontrada implica que, en promedio, no se detecta el $25 \%$ de la carga de infección por SARS-CoV-2 en el país, lo que repercute en la transmisión de la infección en la comunidad. De hecho, si se considerara la sensibilidad $(43,8 \%)$ y especificidad $(98,8 \%)$ de la prueba serológica rápida evaluada en condiciones de campo por el Instituto Nacional de Salud (7), la positividad real sería 32,0\% y la cantidad de falsos negativos se elevaría a 317139 personas, lo que representa el $50 \%$ de la carga de infección por SARSCoV-2 no detectada (material suplementario). Esta elevada falsa negatividad podría ser incluso mayor si el tiempo de retraso entre el inicio de los síntomas y el reporte del resultado de la prueba diagnóstica fuese menor (es decir, un proceso más rápido o eficiente), pues la revisión Cochrane demuestra que durante la primera semana desde el inicio de síntomas 
Matriz de confusión

\begin{tabular}{c|c|c|}
\multicolumn{1}{c}{ COVID-19 } & \multicolumn{2}{c}{ Sano } \\
\cline { 2 - 3 }$(+)$ & $\mathrm{a}$ & $\mathrm{b}$ \\
$\mathrm{nyn}(-)$ & $\mathrm{d}$ & $\mathrm{a}+\mathrm{b}$ \\
$\mathrm{nyy}$ & $\mathrm{c}+\mathrm{d}$ \\
\cline { 2 - 4 } & $\mathrm{c}$ & $\mathrm{b}+\mathrm{d}$ \\
\hline
\end{tabular}

Positividad observada $(\mathrm{Po})=(\mathrm{a}+\mathrm{b}) / \mathrm{n}$

Positividad real $(\operatorname{Pr})=(a+c) / n$

Falsos negativos $(\mathrm{FN})=\mathrm{c}$

Omisión diagnóstica $(O D)=a / c$
Distribución-resumen de resultados

\begin{tabular}{|c|c|c|}
\hline 309635 & 31951 & 341586 \\
\hline 96796 & 1565581 & 1662377 \\
\hline 406431 & 1597532 & 2003963 \\
\hline
\end{tabular}

$$
\begin{aligned}
& \mathrm{Po}=17,0 \% \\
& \mathrm{Pr}=20,3 \% \\
& \mathrm{FN}=96796 \\
& \mathrm{OD}=3,20
\end{aligned}
$$

Figura 1. Matriz de confusión y distribución resumen de resultados en el universo de personas sometidas a una prueba diagnóstica de infección por SARS-CoV-2 en el Perú al 16 de julio de 2020.

de la COVID-19 la sensibilidad de las pruebas diagnósticas rápidas es exigua, pues no supera el $30,1 \%{ }^{(5)}$.

La falta de información detallada sobre los diferentes tipos de pruebas rápidas disponibles en el mercado nacional, su intensidad de uso y validez diagnóstica (sensibilidad y especificidad) - tanto la declarada por el fabricante como la validada en el terreno- es una limitante de la precisión de nuestros estimados de positividad real, falsa negatividad y omisión diagnóstica de la infección por SARS-CoV-2. No obstante, incurrir en tal sesgo potencial de medición tendería a subestimar la verdadera prevalencia, de modo que nuestros estimados pueden considerarse como conservadores.

Las pruebas de laboratorio son elementos esenciales para establecer el diagnóstico certero de la infección por SARSCoV-2 y guiar el curso clínico y tratamiento del paciente, pero también son cruciales para informar sobre la magnitud de la epidemia y la velocidad de su propagación en la población y, por tanto, para guiar el establecimiento e intensidad de las medidas de contención y control epidemiológicos.

$\mathrm{Al}$ igual que la presencia no detectada de personas asintomáticas, la falta de acceso oportuno a diagnóstico confirmatorio, la detectabilidad de anticuerpos dependiente del curso natural de la infección y otros factores relevantes no explorados en esta evaluación que afectan el desempeño de las pruebas diagnósticas, la considerable proporción de resultados falsos negativos aquí documentada y la consecuente subestimación de la real positividad (y prevalencia poblacional) puede distorsionar inadvertida y significativamente la correcta caracterización de la pandemia en el país y explicar la intensa y continua transmisión poblacional a pesar de las tempranas e inéditas medidas de contención epidémica decretadas.
Contribuciones de los autores: OJM y LSO concibieron el artículo, recolectaron e interpretaron datos, hicieron la revisión crítica del artículo y aprobaron su versión final. OJM hizo el análisis cuantitativo de los datos y redactó el artículo.

Financiamiento: Autofinanciado.

Conflictos de interés: Los autores declaran no tener conflictos de interés.

Material suplementario: Disponible en la versión electrónica de la RPMESP.

\section{REFERENCIAS BIBLIOGRÂFICAS}

1. Ministerio de Salud. Comunicado $N^{\circ} 173$. Nota de Prensa: 16 de julio de 2020. Lima: MINSA; 2020. Disponible en: https://www.gob.pe/institucion/ minsa/noticias/212171-minsa-casos-confirmados-por-coronavirus-covid-19-ascienden-a-341-586-en-el-peru-comunicado-n-173.

2. Ahlbom A, Norell S. Introduction to Modern Epidemiology. Chesnut Hill: Epidemiology Resources Inc; 1984.

3. Kim H, Hong H, Yoon S. Diagnostic Performance of CT and Reverse Transcriptase Polymerase Chain Reaction for Coronavirus Disease 2019: A Meta-Analysis. Radiology. 2020;296(3):E145-E155. doi: 10.1148/radiol.2020201343.

4. Zitek T. The appropriate use of testing for COVID-19. West J Emerg Med 2020;21(3):470-472. doi: 10.5811/westjem.2020.4.47370.

5. Deeks JJ, Dinnes J, Takwoingi Y, Davenport C, Spijker R, Taylor-Phillips $\mathrm{S}$, et al. Antibody tests for identification of current and past infection with SARS-CoV-2. Cochrane Database Syst Rev. 2020;6(6):CD013652. doi: 10.1002/14651858.CD013652.

6. Ministerio de Salud. Reporte de Vigilancia COVID-19; Perú, Año 2020. Actualización: 04 Julio. Lima: Centro Nacional de Epidemiología, Prevención y Control de Enfermedades; 2020.

7. Vidal-Anzardo M, Solis G, Solary L, Minaya G, Ayala-Quintanilla B, Astete-Cornejo J, et al. Evaluación en condiciones de campo de una prueba serológica rápida para detección de anticuerpos IgM e IgG contra SARS-CoV-2. Rev Peru Med Exp Salud Publica. 2020;37(2):203-9. doi: 10.17843/rpmesp.2020.372.5534. 